September 3, 2018 7:35 WSPC BaryonicMatter

International Journal of Modern Physics: Conference Series

(C) World Scientific Publishing Company

\title{
Skyrmions, half-skyrmions and nucleon mass in dense baryonic matter
}

\author{
Yong-Liang Ma \\ Department of Physics, Nagoya University, Nagoya, 464-8602, Japan \\ ylma@hken.phys.nagoya-u.ac.jp \\ Masayasu Harada \\ Department of Physics, Nagoya University, Nagoya, 464-8602, Japan \\ harada@hken.phys.nagoya-u.ac.jp \\ Hyun Kyu Lee \\ Department of Physics, Hanyang University, Seoul 133-791, Korea \\ hyunkyu@hanyang.ac.kr \\ Yongseok $\mathrm{Oh}$ \\ Department of Physics, Kyungpook National University, Daegu 702-701, Korea \\ Asia Pacific Center for Theoretical Physics, Pohang, Gyeongbuk 790-784, Korea \\ yohphy@knu.ac.kr \\ Mannque Rho \\ Department of Physics, Hanyang University, Seoul 133-791, Korea \\ Institut de Physique Théorique, CEA Saclay, 91191 Gif-sur-Yvette cédex, France \\ mannque.rho@cea.fr
}

We explore the hadron properties in dense baryonic matter in a unified way by using a Skyrme model constructed with an effective Lagrangian which includes the $\rho$ and $\omega$ vector mesons as hidden gauge bosons and is valid up to $O\left(p^{4}\right)$ in chiral expansion including the homogeneous Wess-Zumino terms. With the two input values of pion decay constant and the lowest lying vector meson mass which can be fixed in free space, all the other low energy constants in the effective Lagrangian are determined by their master formulas derived from holographic QCD models, which allows us to study the baryonic matter properties with no additional free parameters and thus without ambiguities. We find that the $\omega$ field that figures in the homogeneous Wess-Zumino term plays a crucial role in the skyrmion structure and its matter properties. The most striking and intriguing observation is that the pion decay constant that smoothly drops with increasing density in the Skyrmion phase stops decreasing at $n_{1 / 2}$ at which the skyrmions in medium fractionize into half-skyrmions and remains nearly constant in the half-skyrmion phase. In accordance with the large $N_{c}$ consideration, the baryon mass also stays non-scaling in the half-skyrmion phase. This feature is supported by the nuclear effective field theory with the parameters of the Lagrangian scaling modified at the skyrmion-half-skyrmion phase transition. Our exploration also uncovers the crucial role of the $\omega$ meson in multi- 
baryon systems as well as in the structure of a single skyrmion.

Keywords: Skyrme model, hidden local symmetry, nuclear matter, nucleon mass

PACS numbers: 12.39.Dc, 12.39.Fe, 21.65.-f, 21.65.Jk

\section{Introduction}

The physics of baryonic matter poses a challenge in nuclear and particle physics and is crucial to understand the equation of state relevant for compact stars and the QCD phase structure at low temperature. The difficulties in accessing compressed baryonic matter arise from the highly nonperturbative nature of strong interactions, lacking both experimental data and trustful theoretical tools. The situation is acerbated by that QCD lattice simulation suffers from the famous sign problem. As a possible way to access this difficult problem, we apply Skyrme's approach 1 , where the classical soliton solutions of the mesonic theory of QCD are interpreted as baryons in the sense of large $N_{c}$ limit. This model was proposed in Ref. 3 as a natural framework to explore compressed baryonic matter by putting the skyrmions on the crystal lattice 2 . This approach has the merit that allows one to study in a unified way the properties of elementary baryon, many-baryon systems and medium-modified hadron properties.

In pursuance of Skyrme's seminal idea, we explored the nuclear matter and in-medium hadron properties by the hidden local symmetry (HLS) Lagrangian expanded to the chiral order $O\left(p^{4}\right)$ including the lowest lying $\rho$ and $\omega$ vector mesons $4|5| 6$. Relegating details to Refs. 3, 7, 8, 9, 10, we give a brief summary of the main ideas and the main results of our approach. In comparison with what is available in the existing literature, our approach has the following distinctive features:

- We use a chiral effective theory of mesons, which has a self-consistent chiral counting mechanism, including pseudoscalar and lowest lying $\rho$ and $\omega$ vector mesons. The full Lagrangian up to the next to leading order of the chiral counting including the homogeneous Wess-Zumino (hWZ) terms is applied.

- The low energy constants (LECs) of the effective Lagrangian are selfconsistently determined by a set of master formulas derived from a class of holographic QCD (hQCD) models. Therefore, we can fix their values without ambiguity with only two input values of the pion decay constant $f_{\pi}$ and the vector meson mass $m_{\rho}$.

- Using this Lagrangian with the LECs determined as above, we explore the single skyrmion, skyrmion matter, and medium-modified hadron properties. The skyrmion matter is accessed by putting the skyrmions onto the facecentered cubic (FCC) crystal. By regarding mesons as fluctuations with respect to the skyrmion, we can study the in-medium properties of hadrons.

- We include both the isovector $\rho$ meson and isoscalar $\omega$ meson to explore the different roles of these mesons in nuclear matter. 
Using the chiral order $O\left(p^{4}\right)$ HLS Lagrangian we found the followings.

- Compared with the original Skyrme model that includes only pions, the existence of the $\rho$ meson reduces the skyrmion mass and suppresses its radius. However, the $\omega$ meson effect is exactly opposite to that of the $\rho$ meson, i.e., with the existence of the $\omega$ the skyrmion mass is enhanced and its radius increases.

- The vector mesons affect the skyrmion matter properties notably. The $\rho$ meson favors a high value for the half-skyrmion phase transition density $n_{1 / 2}$, while the $\omega$ prefers a smaller value of $n_{1 / 2}$. When the both effects are included $n_{1 / 2}$ becomes similar to the normal nuclear density $n_{0}$.

- In nuclear matter, $f_{\pi}^{*}$ decreases smoothly with increasing density until the density reaches $n_{1 / 2}$ and after that it becomes more or less a nonzero constant. Moreover, the in-medium nucleon mass $m_{N}^{*}$ also has a similar density-dependence. These similarities are supported by the scaling of $m_{N} \propto f_{\pi} / e \sim\left(\sqrt{N_{c}}\right)^{2}$ in the Skyrme model where the Skyrme parameter $e$ is $O\left(1 / \sqrt{N_{c}}\right)$.

The features of our approach summarized above will be discussed in more detail in the following manner. We begin, in Sec. 2, with providing the HLS Lagrangian that is the building block of our model for the structure and matter properties of skyrmions. We also introduce the master formulas for the LECs based on hQCD. Then the skyrmion properties are explored in this approach and the skyrmion matter properties are studied by putting the skyrmions on the FCC crystal in Sec. 3. Special emphasis is put on the role of vector mesons on the value of $n_{1 / 2}$, where the halfskyrmion phase appears. In Sec. 4 we discuss the hadron properties in nuclear medium, and summarize the results and discussions in Sec. 5 .

\section{Baryon properties in hidden local symmetry}

The HLS Lagrangian under consideration for investigating the structure and matter properties of skyrmions has the symmetry group $G_{\text {full }}=\left[\mathrm{SU}(2)_{L} \times \mathrm{SU}(2)_{R}\right]_{\text {chiral }} \times$ $[\mathrm{U}(2)]_{\mathrm{HLS}}$. Here, $[\mathrm{U}(2)]_{\mathrm{HLS}}$ is adopted as the "hidden local symmetry" to incorporate the $\rho$ and $\omega$ vector mesons. The basic building blocks of HLS Lagrangian are two 1 -forms $\hat{\alpha}_{\| \mu}$ and $\hat{\alpha}_{\perp \mu}$ defined by

$$
\hat{\alpha}_{\| \mu}=\frac{1}{2 i}\left(D_{\mu} \xi_{R} \xi_{R}^{\dagger}+D_{\mu} \xi_{L} \xi_{L}^{\dagger}\right), \quad \hat{\alpha}_{\perp \mu}=\frac{1}{2 i}\left(D_{\mu} \xi_{R} \xi_{R}^{\dagger}-D_{\mu} \xi_{L} \xi_{L}^{\dagger}\right),
$$

with the chiral fields $\xi_{L}$ and $\xi_{R}$, which are expressed in the unitary gauge as

$$
\xi_{L}^{\dagger}=\xi_{R}=e^{i \pi / 2 f_{\pi}} \equiv \xi \quad \text { with } \quad \pi=\pi \cdot \tau / 2,
$$

where $\boldsymbol{\tau}$ 's are the Pauli matrices. The covariant derivative is defined as

$$
D_{\mu} \xi_{R, L}=\left(\partial_{\mu}-i V_{\mu}\right) \xi_{R, L}
$$

where $V_{\mu}=\frac{g}{2}\left(\omega_{\mu}+\rho_{\mu}\right)$ is the gauge boson of the HLS with $\rho_{\mu}=\boldsymbol{\rho}_{\mu} \cdot \boldsymbol{\tau}$. 
Then the HLS Lagrangian adopted in this work can be written as

$$
\mathcal{L}_{\mathrm{HLS}}=\mathcal{L}_{(2)}+\mathcal{L}_{(4)}+\mathcal{L}_{\text {anom }}
$$

The chiral order $O\left(p^{2}\right)$ term, $\mathcal{L}_{(2)}$, is constructed as $\frac{4516}{46}$

$$
\mathcal{L}_{(2)}=f_{\pi}^{2} \operatorname{Tr}\left(\hat{\alpha}_{\perp \mu} \hat{\alpha}_{\perp}^{\mu}\right)+a f_{\pi}^{2} \operatorname{Tr}\left(\hat{\alpha}_{\| \mu} \hat{\alpha}_{\|}^{\mu}\right)-\frac{1}{2 g^{2}} \operatorname{Tr}\left(V_{\mu \nu} V^{\mu \nu}\right),
$$

where $f_{\pi}$ is the pion decay constant, $a$ is the HLS parameter, $g$ is the vector meson coupling constant, and the field-strength tensor of the vector meson is $V_{\mu \nu}=\partial_{\mu} V_{\nu}-$ $\partial_{\nu} V_{\mu}-i\left[V_{\mu}, V_{\nu}\right]$. The $O\left(p^{4}\right)$ Lagrangian at the leading order in $N_{c}$ is given by

$$
\begin{aligned}
\mathcal{L}_{(4)}= & \mathcal{L}_{(4) y}+\mathcal{L}_{(4) z}, \\
\mathcal{L}_{(4) y}= & y_{1} \operatorname{Tr}\left[\hat{\alpha}_{\perp \mu} \hat{\alpha}_{\perp}^{\mu} \hat{\alpha}_{\perp \nu} \hat{\alpha}_{\perp}^{\nu}\right]+y_{2} \operatorname{Tr}\left[\hat{\alpha}_{\perp \mu} \hat{\alpha}_{\perp \nu} \hat{\alpha}_{\perp}^{\mu} \hat{\alpha}_{\perp}^{\nu}\right] \\
& +y_{3} \operatorname{Tr}\left[\hat{\alpha}_{\| \mu} \hat{\alpha}_{\|}^{\mu} \hat{\alpha}_{\| \nu} \hat{\alpha}_{\|}^{\nu}\right]+y_{4} \operatorname{Tr}\left[\hat{\alpha}_{\| \mu} \hat{\alpha}_{\| \nu} \hat{\alpha}_{\|}^{\mu} \hat{\alpha}_{\|}^{\nu}\right] \\
& +y_{5} \operatorname{Tr}\left[\hat{\alpha}_{\perp \mu} \hat{\alpha}_{\perp}^{\mu} \hat{\alpha}_{\| \nu} \hat{\alpha}_{\|}^{\nu}\right]+y_{6} \operatorname{Tr}\left[\hat{\alpha}_{\perp \mu} \hat{\alpha}_{\perp \nu} \hat{\alpha}_{\|}^{\mu} \hat{\alpha}_{\|}^{\nu}\right]+y_{7} \operatorname{Tr}\left[\hat{\alpha}_{\perp \mu} \hat{\alpha}_{\perp \nu} \hat{\alpha}_{\|}^{\nu} \hat{\alpha}_{\|}^{\mu}\right] \\
& +y_{8}\left\{\operatorname{Tr}\left[\hat{\alpha}_{\perp \mu} \hat{\alpha}_{\|}^{\mu} \hat{\alpha}_{\perp \nu} \hat{\alpha}_{\|}^{\nu}\right]+\operatorname{Tr}\left[\hat{\alpha}_{\perp \mu} \hat{\alpha}_{\| \nu} \hat{\alpha}_{\perp}^{\nu} \hat{\alpha}_{\|}^{\mu}\right]\right\}+y_{9} \operatorname{Tr}\left[\hat{\alpha}_{\perp \mu} \hat{\alpha}_{\| \nu} \hat{\alpha}_{\perp}^{\mu} \hat{\alpha}_{\|}^{\nu}\right], \\
\mathcal{L}_{(4) z}= & i z_{4} \operatorname{Tr}\left[V_{\mu \nu} \hat{\alpha}_{\perp}^{\mu} \hat{\alpha}_{\perp}^{\nu}\right]+i z_{5} \operatorname{Tr}\left[V_{\mu \nu} \hat{\alpha}_{\|}^{\mu} \hat{\alpha}_{\|}^{\nu}\right] .
\end{aligned}
$$

Finally, the anomalous-parity homogeneous WZ (hWZ) terms are written as

$$
\Gamma_{\mathrm{hWZ}}=\int d^{4} x \mathcal{L}_{\text {anom }}=\frac{N_{c}}{16 \pi^{2}} \int_{M^{4}} \sum_{i=1}^{3} c_{i} \mathcal{L}_{i},
$$

where $M^{4}$ stands for the four-dimensional Minkowski space and

$$
\begin{aligned}
& \mathcal{L}_{1}=i \operatorname{Tr}\left[\hat{\alpha}_{\mathrm{L}}^{3} \hat{\alpha}_{\mathrm{R}}-\hat{\alpha}_{\mathrm{R}}^{3} \hat{\alpha}_{\mathrm{L}}\right], \quad \mathcal{L}_{2}=i \operatorname{Tr}\left[\hat{\alpha}_{\mathrm{L}} \hat{\alpha}_{\mathrm{R}} \hat{\alpha}_{\mathrm{L}} \hat{\alpha}_{\mathrm{R}}\right], \\
& \mathcal{L}_{3}=\operatorname{Tr}\left[F_{\mathrm{V}}\left(\hat{\alpha}_{\mathrm{L}} \hat{\alpha}_{\mathrm{R}}-\hat{\alpha}_{\mathrm{R}} \hat{\alpha}_{\mathrm{L}}\right)\right],
\end{aligned}
$$

in the 1-form and 2-form notations with

$$
\hat{\alpha}_{L}=\hat{\alpha}_{\|}-\hat{\alpha}_{\perp}, \quad \hat{\alpha}_{R}=\hat{\alpha}_{\|}+\hat{\alpha}_{\perp}, \quad F_{V}=d V-i V^{2} .
$$

The low energy constants $\left(f_{\pi}, a, g, y_{i}, z_{i} c_{i}\right)$ of the Lagrangian can be calculated by using the general "master formula" proposed in Ref. 11 from a class of holographic QCD models, namely,

$$
\begin{aligned}
& f_{\pi}^{2}=N_{c} G_{\mathrm{YM}} M_{K K}^{2} \int d z K_{2}(z)\left[\dot{\psi}_{0}(z)\right]^{2}, \quad a f_{\pi}^{2}=N_{c} G_{\mathrm{YM}} M_{K K}^{2} \lambda_{1}\left\langle\psi_{1}^{2}\right\rangle, \\
& \frac{1}{g^{2}}=N_{c} G_{\mathrm{YM}}\left\langle\psi_{1}^{2}\right\rangle, \quad y_{1}=-y_{2}=-N_{c} G_{\mathrm{YM}}\left\langle\left(1+\psi_{1}-\psi_{0}^{2}\right)^{2}\right\rangle, \\
& y_{3}=-y_{4}=-N_{c} G_{\mathrm{YM}}\left\langle\psi_{1}^{2}\left(1+\psi_{1}\right)^{2}\right\rangle, \quad y_{5}=2 y_{8}=-y_{9}=-2 N_{c} G_{\mathrm{YM}}\left\langle\psi_{1}^{2} \psi_{0}^{2}\right\rangle, \\
& y_{6}=-\left(y_{5}+y_{7}\right), \quad y_{7}=2 N_{c} G_{\mathrm{YM}}\left\langle\psi_{1}\left(1+\psi_{1}\right)\left(1+\psi_{1}-\psi_{0}^{2}\right)\right\rangle, \\
& z_{4}=2 N_{c} G_{\mathrm{YM}}\left\langle\psi_{1}\left(1+\psi_{1}-\psi_{0}^{2}\right)\right\rangle, \quad z_{5}=-2 N_{c} G_{\mathrm{YM}}\left\langle\psi_{1}^{2}\left(1+\psi_{1}\right)\right\rangle,
\end{aligned}
$$


Table 1. Skyrmion mass and size obtained in models of the HLS Lagrangian.

\begin{tabular}{ccccc}
\hline & $\operatorname{HLS}(\pi, \rho, \omega)$ & $\operatorname{HLS}(\pi, \rho)$ & $\operatorname{HLS}(\pi)$ & $\operatorname{HLS}_{\min }(\pi, \rho, \omega)$ \\
\hline$M_{\mathrm{sol}}(\mathrm{MeV})$ & 1184 & 834 & 922 & 1407 \\
$\Delta_{M}(\mathrm{MeV})$ & 448 & 1707 & 1014 & 259 \\
\hline$\sqrt{\left\langle r^{2}\right\rangle_{W}}(\mathrm{fm})$ & 0.433 & 0.247 & 0.309 & 0.540 \\
$\sqrt{\left\langle r^{2}\right\rangle_{E}}(\mathrm{fm})$ & 0.608 & 0.371 & 0.417 & 0.725 \\
\hline
\end{tabular}

$$
\begin{aligned}
& c_{1}=\left\langle\left\langle\dot{\psi}_{0} \psi_{1}\left(\frac{1}{2} \psi_{0}^{2}+\frac{1}{6} \psi_{1}^{2}-\frac{1}{2}\right)\right\rangle\right\rangle, \\
& c_{2}=\left\langle\left\langle\dot{\psi}_{0} \psi_{1}\left(-\frac{1}{2} \psi_{0}^{2}+\frac{1}{6} \psi_{1}^{2}+\frac{1}{2} \psi_{1}+\frac{1}{2}\right)\right\rangle\right\rangle, \quad c_{3}=\left\langle\left\langle\frac{1}{2} \dot{\psi}_{0} \psi_{1}^{2}\right\rangle\right\rangle,
\end{aligned}
$$

where the smallest nonzero eigenvalue $\lambda_{1}$ and its corresponding eigenfunction $\psi_{1}(z)$ satisfy the eigenvalue equation (with $n=1$ )

$$
-K_{1}^{-1}(z) \partial_{z}\left[K_{2}(z) \partial_{z} \psi_{n}(z)\right]=\lambda_{n} \psi_{n}(z),
$$

with $K_{1}(z)$ and $K_{2}(z)$ being the warping factors of the 5-dimensional space-time. In Eq. (10) we made use of the following definitions:

$$
\langle A\rangle \equiv \int_{-\infty}^{\infty} d z K_{1}(z) A(z), \quad\langle\langle A\rangle\rangle \equiv \int_{-\infty}^{\infty} d z A(z) .
$$

Here, we take $K_{1}(z)=K^{-1 / 3}(z)$ and $K_{2}(z)=K(z)$ with $K(z)=1+z^{2}$ corre-

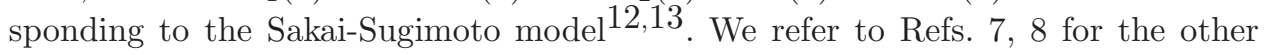
choices.

Thanks to the master formulas, once the hQCD parameters $M_{\mathrm{KK}}$ and $G_{\mathrm{YM}}$ are determined by, for example, the empirical values of $f_{\pi}$ and $m_{\rho}$, all the rest of the parameters of the effective Lagrangian except the HLS parameter $a$ are fixed. In this work, we use

$$
f_{\pi}=92.4 \mathrm{MeV}, \quad m_{\rho}=775.5 \mathrm{MeV} .
$$

Armed with the master formulas and the input values, we now study the single soliton properties. The purpose of this study is to find the qualitatively robust features of the soliton structure rather than quantitative agreements with nature. Our results of soliton mass $M_{\text {sol }}, \Delta-N$ mass splitting $\Delta_{M}$, the winding number radius $\sqrt{\left\langle r^{2}\right\rangle_{W}}$, and the energy radius $\sqrt{\left\langle r^{2}\right\rangle_{E}}$ are summarized in Table 1 . Here, we consider three versions of the HLS Lagrangian. The full version $\operatorname{HLS}(\pi, \rho, \omega)$ includes the pion, $\rho$, and $\omega$ mesons. In order to see the role of the $\omega$ meson, we consider $\operatorname{HLS}(\pi, \rho)$ that does not have th $\omega$ meson, which can be achieved by discarding the hWZ terms. Finally the role of the $\rho$ meson can be studied by comparing the results of the model $\operatorname{HLS}(\pi)$ that is obtained by integrating out the $\rho$ field in $\operatorname{HLS}(\pi, \rho)$. For comparison, we also list the results of so-called "the minimal model" 14 that corresponds to setting $y_{i}=z_{i}=c_{3}=0$ and $c_{1}=-c_{2}=2 / 3$ in Eq. (4). The details can be found in Ref. 8 . 


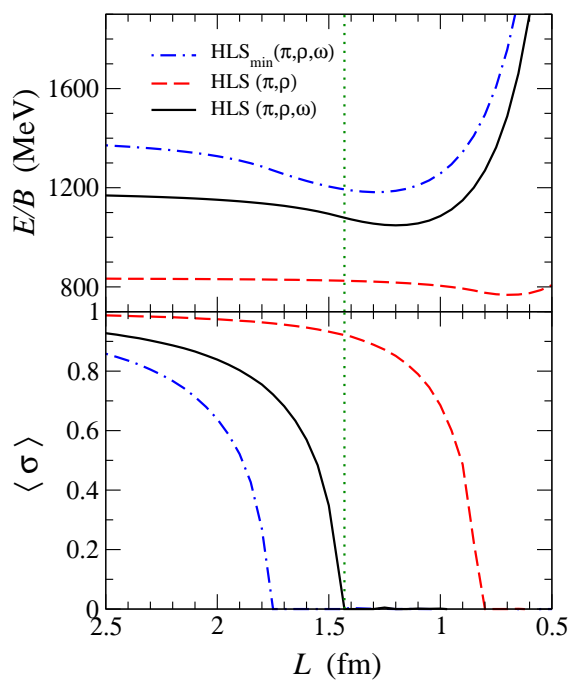

Fig. 1. $E / B$ and $\langle\sigma\rangle$ of the minimum energy configuration for a given $L$.

Table1 1 shows explicitly the role of the vector mesons in the skyrmion properties. The $\rho$ meson reduces the soliton mass from $922 \mathrm{MeV}$ in $\operatorname{HLS}(\pi)$ to $834 \mathrm{MeV}$ in $\operatorname{HLS}(\pi, \rho)$, but the $\omega$ meson restores the large soliton mass by giving $1184 \mathrm{MeV}$ in $\operatorname{HLS}(\pi, \rho, \omega)$. These tendency can also be found in the skyrmion radii. However, the $\Delta-N$ mass difference, which is related to the moment of inertia of the soliton, shows the opposite. Without the $\omega$ meson, this mass difference becomes vary large and breaks the standard quantization method of skyrmions. This emphasizes the important role driven by the $\omega$ meson.

\section{Dense baryonic matter from HLS}

The dense baryonic matter can be simulated by putting skyrmions obtained in this model onto the FCC crystal. For studying the baryonic matter in large $N_{c}$ limit, we approximate the baryonic matter by the skyrmion matter. The baryonic matter density $\rho$ is controlled by the crystal size $2 L$ as $\rho \propto 1 / L^{3}$.

Our results for the dependence of per-skyrmion energy $E / B$ and $\langle\sigma\rangle$ on the FCC size parameter $L$ are shown in Fig. 1. Here, $\langle\sigma\rangle$ is the space average of the chiral field $U$ over the space that a single skyrmion occupies and it vanishes in the half-skyrmion phase. The results for $\operatorname{HLS}(\pi, \rho, \omega), \operatorname{HLS}(\pi, \rho)$ and $\operatorname{HLS}_{\min }(\pi, \rho, \omega)$ are illustrated by solid, dashed, and dash-dotted lines, respectively. The position of the normal nuclear matter density is denoted by a vertical dotted line.

Figure 1 suggests that, in $\operatorname{HLS}(\pi, \rho, \omega), n_{1 / 2} \cong n_{0}$. Comparing to the results of $\operatorname{HLS}_{\min }(\pi, \rho, \omega)$, the $O\left(p^{4}\right)$ terms and the other $\pi-\rho$ - $\omega$ interactions through the $\mathrm{hWZ}$ terms make $n_{1 / 2}$ larger by a factor 1.7 . This higher value of $n_{1 / 2}$ comes from the fact that the size of the single skyrmion is smaller in the former and that the 
additional interactions in $\operatorname{HLS}(\pi, \rho, \omega)$ weaken the repulsive interactions from the $\omega$. This conclusion is supported by the results from $\operatorname{HLS}(\pi, \rho)$, where $n_{1 / 2} \cong 6 n_{0}$. This means that the absence of the $\omega$ meson reduces the skyrmion size to almost half compared to that of $\operatorname{HLS}(\pi, \rho, \omega)$. Moreover, the weak dependence of $E / B$ on density in $\operatorname{HLS}(\pi, \rho)$ is noticeable, which indicates that the inclusion of the $\rho$ meson reduces the soliton mass and almost saturates the Bogomol'nyi bound.

\section{Hadron properties with the FCC crystal background}

When the meson fields in the Lagrangian is expanded in terms of the fluctuating meson fields about their vacuum values, the meson dynamics can be investigated in free space. By the same way, if we incorporate the fluctuations with respect to classical solutions for $U, \rho_{\mu}^{a}$, and $\omega_{\mu}$ discussed in the previous section, these fields describe the corresponding mesons and their dynamics in dense baryonic matter. We denote the minimum energy solutions as $U_{(0)}=\xi_{(0) L}^{\dagger} \xi_{(0) R}, \rho_{\mu}^{a(0)}$, and $\omega_{\mu}^{(0)}$, and introduce the fluctuating fields on top of the classical solutions as

$$
\xi_{L, R}=\xi_{(0) L, R} \tilde{\xi}_{L, R}, \quad \rho_{\mu}^{a}=\rho_{\mu}^{a(0)}+\tilde{\rho}_{\mu}^{a}, \quad \omega_{\mu}=\omega_{\mu}^{(0)}+\tilde{\omega}_{\mu},
$$

where $\tilde{\xi}_{L}^{\dagger}=\tilde{\xi}_{R}=\tilde{\xi}=\exp \left(i \tau_{a} \tilde{\pi}_{a} / 2 f_{\pi}\right)$, $\tilde{\rho}_{\mu}^{a}$, and $\tilde{\omega}_{\mu}$ stand for the corresponding fluctuating fields. By substituting these fields into the HLS Lagrangian and taking the space average for the background field configurations as denoted by $\langle\cdot\rangle$, we can find that the pion kinetic term is modified as

$$
\mathcal{L}=\frac{1}{2}\left[1-\frac{2}{3}\left(1-\left\langle\sigma_{(0)}^{2}\right\rangle\right)\right] \partial_{\mu} \pi^{a} \partial^{\mu} \pi^{a},
$$

where we have taken into account only the first term of the HLS Lagrangian since at densities away from that of chiral restoration with which we are concerned here the other terms represent effectively higher order in the chiral counting. The extra factor in front of the pion kinetic term (15) suggests that the in-medium pion decay constant $f_{\pi}^{*}$ reads

$$
\frac{f_{\pi}^{*}}{f_{\pi}}=\sqrt{1-\frac{2}{3}\left(1-\left\langle\sigma_{(0)}^{2}\right\rangle\right)} .
$$

In the left panel of Fig. 2, we present $f_{\pi}^{*} / f_{\pi}$ as a function of the FCC box size. This shows that the density dependence of $f_{\pi}^{*} / f_{\pi}$ in the single-skyrmion phase is very different from that in the half-skyrmion phase. In the single-skyrmion phase, $f_{\pi}^{*} / f_{\pi}$ decreases as the baryon number density increases. In the half-skyrmion phase, however, it almost stays at a non-vanishing value of $\sim 0.65$. Since $f_{\pi}^{*}$ does not vanish, the half-skyrmion phase was interpreted as a sort of pseudo-gap phase in Ref. 15.

In order to determine the LECs of our effective Lagrangian we made use of the master formulas. As the simplest approximation in applying it to dense matter, we assume that the in-medium modification of all the LECs can be calculated using the in-medium values $f_{\pi}^{*}$ and $m_{\rho}^{*}$ which renders us to explore the medium modified baryon mass. With this approximation the in-medium single-skyrmion mass can be 

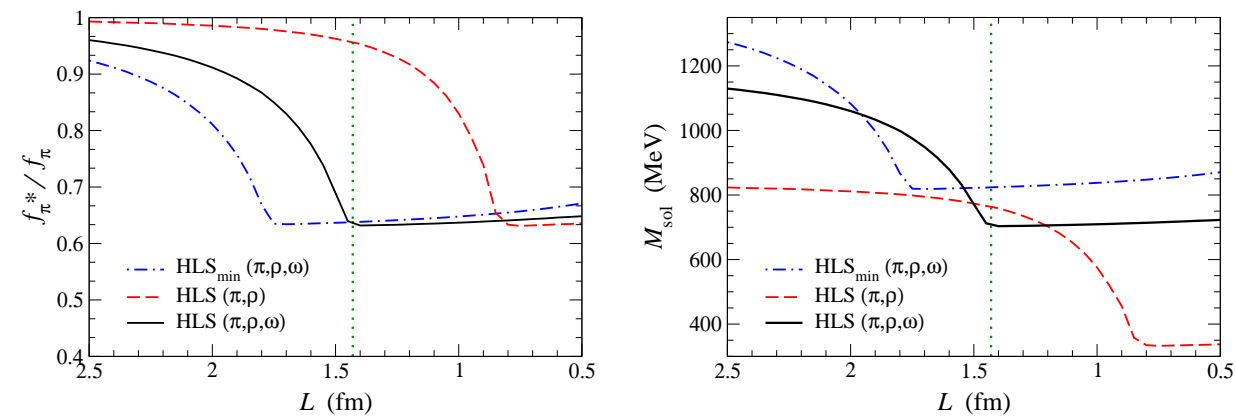

Fig. 2. Crystal size dependence of $f_{\pi}^{*} / f_{\pi}$ and $M_{\text {sol }}$ with the FCC crystal background.

calculated and the results are hown in the right panel of Fig. 2, This shows that the soliton mass $M_{\mathrm{sol}}^{*}$ has a nearly identical density dependence as $f_{\pi}^{*}$. This feature can be understood by the fact that in the skyrmion model the soliton mass scales roughly as $M_{\mathrm{sol}}^{*} \sim f_{\pi}^{*} / e$ where $e$ is the Skyrme parameter, which follows from the scaling of the nucleon mass in the large $N_{c}$ limit as in matter-free space.

Our principal finding in this calculation is that the nucleon mass, which decreases with increasing density in the skyrmion phase, stops dropping at $n_{1 / 2}$ and stays nearly constant during the half-skyrmion phase. In the case of $\operatorname{HLS}(\pi, \rho, \omega)$, the in-medium soliton mass approaches $\sim 0.6 M_{\text {sol }}$. In the skyrmion phase with $n<$ $n_{1 / 2}$, chiral symmetry is broken with the order parameter $\langle\bar{q} q\rangle \neq 0$ and hadrons are massive apart from the Nambu-Goldstone bosons, i.e., the pions. In the halfskyrmion phase, we have $\langle\bar{q} q\rangle=0$ on the average, but chiral symmetry is not restored yet since hadrons are still massive and there exist pions. There seems to be no obvious order parameter characterizing this state apart from the presence of the half-skyrmion structure arising from a topology change. The nucleon mass staying as a constant in the half-skyrmion phase indicates the origin of the nucleon mass. What this means is that the nucleon mass could have a component that remains non-vanishing up to the chiral transition, which is reminiscent of the parity doublet picture of baryons 16 , where the nucleon mass is given by

$$
m_{N}=m_{0}+\Delta(\langle\bar{q} q\rangle)
$$

where $m_{0}$ is the chiral invariant mass and $\Delta$ is the part of the mass that vanishes as $\langle\bar{q} q\rangle \rightarrow 0$ (in the chiral limit) for $n \rightarrow n_{c}$. From $M_{\text {sol }}$ illustated in Fig. 2 one can imagine the crystal size dependence of the part of the nucleon mass originning from the chiral symmetry breaking.

\section{Summary and discussions}

In a series of publications, we have explored to unravel the structure of baryonic matter at high density exploiting Skyrme's conjecture. Our study includes the lowest lying vector mesons $\rho$ and $\omega$ in the the HLS approach with the Lagrangian given up 
to $O\left(p^{4}\right)$ in chiral counting and the leading order in $N_{c}$. The LECs of the Lagrangian are then determined by using the master formulas derived from a class of hQCD models.

Our results clearly show that the skyrmion and skyrmion matter properties are affected by the attractive interaction of the $\rho$ meson and the repulsive interaction induced by the $\omega$ meson. We confirm the observation made by Sutcliffe 17 that the incorporation of the tower of isovector vector mesons brings the soliton mass close to the BPS bound, where the lowest $\rho$ vector meson plays a striking role. As stressed by Sutcliffe, this approach to the BPS state would ameliorate the binding energy structure of heavy nuclei, which the Skyrme model fails to describe. What is found in this work is that the presence of the isoscalar vector meson $\omega$ obstructs this approach to the BPS state. Whether the inclusion of the infinite tower of $\omega$ 's as implied in hQCD will change this feature is not known at the moment.

What is intriguing is that independently of how the BPS state is approached, the Bethe-Weizsäcker mass formula is very well reproduced by a BPS model as shown recently in Ref. 18. This model is anchored on self-dual configurations with no interactions. The small binding energy observed is then given by a small perturbation. This picture is diagonally opposed to the standard nuclear physics lore where the small binding energy is given by a near complete cancellation between two large quantities, an attractive one and a repulsive one. Whether there is a "dual" relation between the two opposed pictures is an intriguing issue.

On the crystal lattice, we find that the nucleon mass decreases smoothly as density increases up to $n_{1 / 2}$ and, in the half-skyrmion phase, the dropping of the nucleon mass stops and the mass remains constant going toward to the chiral restoration. In the skyrmion phase with density $n<n_{1 / 2}$, chiral symmetry is broken with the order parameter $\langle\bar{q} q\rangle \neq 0$ and hadrons are massive except the pions. However, in the half-skrymion phase, even with $\langle\bar{q} q\rangle=0$ on the average, chiral symmetry is not restored since hadrons are still massive and there exist pions. That the nucleon mass remains non-vanishing as one approaches the chiral transition point is supported by other approaches. As mentioned, this feature is reminiscent of the parity-doublet baryon model with a substantially large $m_{0}$. Furthermore there is an indication from an extended QCD with a scalar field introduced as an auxiliary field 19 that the structure of nucleons could be basically different from that of mesons as in the chiral bag mode 20 .

It should be noted that, some of our results, such as the nucleon mass, the density $n_{\text {min }}$ at which per-baryon mass is at its minimum, the nucleon binding energy and so on, deviate from nature. This drawback indicates that in our approach some other effects, for example the dilaton and irreducible three-body interactions, are missing. 


\section{Acknowledgments}

The work of Y.-L.M. and M.H. was supported in part by Grant-in-Aid for Scientific Research on Innovative Areas (No. 2104) "Quest on New Hadrons with Variety of Flavors" from MEXT. Y.-L.M. was supported in part by the National Science Foundation of China (NSFC) under Grant No. 10905060. The work of M.H. was supported in part by the Grant-in-Aid for Nagoya University Global COE Program "Quest for Fundamental Principles in the Universe: From Particles to the Solar System and the Cosmos" from MEXT, the JSPS Grant-in-Aid for Scientific Research (S) No. 22224003 and (c) No. 24540266. The work of H.K.L. and M.R. was partially supported by the WCU project of Korean Ministry of Education, Science and Technology (R33-2008-000-10087-0). Y.O. was supported in part by the Basic Science Research Program through the National Research Foundation of Korea under Grant No. NRF-2013R1A1A2A10007294 and B.-Y.P. was supported by the research fund of Chungnam National University.

\section{References}

1. T. H. R. Skyrme, Nucl. Phys. 31, 556 (1962).

2. B.-Y. Park and V. Vento, Skyrmion approach to finite density and temperature, in The Multifaceted Skyrmions, edited by G. E. Brown and M. Rho, World Scientific, Singapore, 2010, arXiv:0906.3263

3. H.-J. Lee, B.-Y. Park, D.-P. Min, M. Rho, and V. Vento, Nucl. Phys. A 723, 427 (2003).

4. M. Bando, T. Kugo, S. Uehara, K. Yamawaki, and T. Yanagida, Phys. Rev. Lett. 54, $1215(1985)$

5. M. Bando, T. Kugo, and K. Yamawaki, Phys. Rep. 164, 217 (1988).

6. M. Harada and K. Yamawaki, Phys. Rep. 381, 1 (2003).

7. Y.-L. Ma, Y. Oh, G.-S. Yang, M. Harada, H. K. Lee, B.-Y. Park, and M. Rho, Phys. Rev. D 86, 074025 (2012).

8. Y.-L. Ma, G.-S. Yang, Y. Oh, and M. Harada, Phys. Rev. D 87, 034023 (2013).

9. Y.-L. Ma, G.-S. Yang, Y. Oh, M. Harada, H.-K. Lee, B.-Y. Park, and M. Rho, talk presented at the workshop on Strong Coupling Gauge Theories in the LHC Perspective, Nagoya, Japan, Dec. 4-7, 2012, arXiv:1304.0289

10. Y.-L. Ma, M. Harada, H. K. Lee, Y. Oh, B.-Y. Park, and M. Rho, Phys. Rev. D 88, 014016 (2013)

11. M. Harada, S. Matsuzaki, and K. Yamawaki, Phys. Rev. D 74, 076004 (2006).

12. T. Sakai and S. Sugimoto, Prog. Theor. Phys. 113, 843 (2005).

13. T. Sakai and S. Sugimoto, Prog. Theor. Phys. 114, 1083 (2005).

14. B.-Y. Park, M. Rho, and V. Vento, Nucl. Phys. A 736, 129 (2004).

15. H.-J. Lee, B.-Y. Park, M. Rho, and V. Vento, Nucl. Phys. A 741, 161 (2004).

16. C. E. Detar and T. Kunihiro, Phys. Rev. D 39, 2805 (1989).

17. P. Sutcliffe, J. High Energy Phys. 1104, 045 (2011).

18. C. Adam, C. Naya, J. Sanchez-Guillen, and A. Wereszczynski, Phys. Rev. Lett. 111, $232501(2013)$

19. D. B. Kaplan, arXiv:1306.5818

20. M. Rho, Phys. Rep. 240, 1 (1994), revised in hep-ph/0206003 